\title{
Língua, cultura e cognição: um estudo do ato de fala do pedido em italiano, português brasileiro, espanhol argentino e alemão
}

\author{
Elisabetta Santoro ${ }^{1}$ \\ Adriana Mendes Porcellato ${ }^{2}$
}

Resumo: Partindo do pressuposto de que a língua é o produto da atividade cognitiva humana que remete a esquemas linguísticos e culturais, buscamos neste estudo verificar como se manifestam linguisticamente essas categorias, analisando o ato de fala do pedido em italiano, português brasileiro, espanhol argentino e alemão no marco da Pragmática cross-cultural. Em especial, concentramos nossa análise na (in)diretividade e na convencionalidade dos atos principais (head acts) e, paralelamente, na perspectiva, visando a entender se os pedidos são orientados pelo falante $(e u)$, pelo interlocutor $(t u)$, pelo nós inclusivo ou ainda por formulações impessoais (BLUM-KULKA et al., 1989). Na linha dos estudos da área, os dados foram coletados com um Discourse Completion Task (DCT), manipulando as variáveis grau de imposição e distância social de oito situações diferentes. Nossa hipótese era que, observando os pedidos desse ponto de vista, poderíamos verificar a presença de convergências e divergências nos traços linguístico-culturais. Embora tenha sido constatada, nas quatro línguas, a predominância de atos principais convencionalmente indiretos e da perspectiva $t u$, há diferenças na distribuição de (in)diretividade e perspectivas que podem indicar peculiaridades na forma como cada cultura percebe categorias como a "invasão" do espaço do outro e a solidariedade.

Palavras-chave: Pragmática cross-cultural. Pedidos. Línguas românicas. Alemão.

Abstract: Starting from the premise that language is a product of human cognition encompassing linguistic and cultural schemata, in this study we set out to understand how these categories emerge from language, by analysing requests in Italian, Brazilian Portuguese, Argentinian Spanish and German from a cross-cultural pragmatic perspective. In particular, we focused our analyses on how (in)directness and conventionality were linguistically realized in head acts and, concurrently, on which perspectives were used, namely whether requests were speaker-oriented, hearer-

\footnotetext{
${ }^{1}$ Departamento de Letras Modernas, PPG em Língua, Literatura e Cultura Italianas, Faculdade de Filosofia, Letras e Ciência Humanas, USP, São Paulo (SP), Brasil - esantoro@usp.br ${ }^{2}$ Doutora pelo PPG em Língua, Literatura e Cultura Italianas, USP - adriana.porcellato@outlook.com
} 


\section{PERcursos Linguísticos • Vitória (ES) •v. 10 •n. 26・2020 • ISSN: 2236-2592 • Dossiê: Linguagem, Comunicação e Cognição •}

oriented, inclusive or impersonal (BLUM-KULKA; HOUSE; KASPER, 1989). In line with studies in this field, data were collected with a Discourse Completion Task (DCT), manipulating the variables rank of imposition and social distance of eight different situations. We hypothesized that, by observing requests from this perspective, we could verify the presence of analogies and differences in linguistic and cultural traits. While we observed a predominance of conventionally indirect head acts and the hearer-oriented perspective in the four languages, there also emerged differences in the distribution of (in)directness and perspectives that can point to peculiarities in how each culture perceives categories such as territory "invasion" and solidarity.

Keywords: Cross-cultural pragmatics. Requests. Romance languages. German.

\section{Introdução}

De acordo com o antropólogo Edward Sapir, a língua pode ser considerada " $a$ symbolic guide to culture" (SAPIR, 1949, p. 162), isto é, uma maneira de entrar em contato com as culturas e de interpretá-las. Observar a(s) língua(s) pode, portanto, oferecer um canal de acesso para o estudo de uma ou mais culturas e ser especialmente relevante na perspectiva cross-cultural, quando o objetivo é identificar analogias e diferenças nos modelos, hábitos e normas específicos de comunicação, que definem cada comunidade de fala e influenciam a maneira como seus membros pensam, se expressam, se comportam e julgam comportamentos.

A fim de investigar esses fenômenos, uma grande variedade de trabalhos dedicou-se a analisar a variação pragmática entre línguas e culturas, dando origem à dita Pragmática cross-cultural ou contrastiva, que se ocupa de realizar "synchronic comparisons between two, or a small number of co-existent language usage systems" (VERSCHUEREN, 2016, p. 3). Esses estudos concentram-se, em sua grande maioria, nos atos de fala, que são considerados, retomando Searle, "basic or minimal units of linguistic communication” (1969, p. 16). O grande interesse pelos atos de fala se deve principalmente ao fato de sua realização ser fortemente influenciada por normas socioculturais, que variam em cada comunidade de fala (BLUM-KULKA; HOUSE; KASPER, 1989) e podem, portanto, ser entendidas como indícios, por meio dos quais é possível identificar como se configura a linguagem enquanto atividade cognitiva, 


\title{
PERcursos Linguísticos • Vitória (ES) •v. $10 \bullet n .26 \bullet 2020 ・$ ISSN: 2236-2592 • Dossiê: Linguagem, Comunicação e Cognição •
}

portadora de referências culturais, de modo que, ao comparar as realizações dos atos de fala, estamos também buscando entender os padrões que regem essas escolhas. Em outras palavras, estudos empíricos sobre a realização dos atos de fala podem nos ajudar a entender o funcionamento das línguas e sua relação com esquemas culturais, sem cair em estereótipos ou simplificações, já que, como lembra Leech:

\begin{abstract}
Statements such as "the Japanese are more modest than the British" or "the British are more tactful than the Americans" only make sense if we relativise them to pragmalinguistic strategies such as strategies of indirectness, and the norms observed in the performance of these strategies in different speech communities. (1983, p. 281)
\end{abstract}

É preciso, contudo, levar em conta que os estudos com comparações entre duas línguas não são considerados suficientes para identificar padrões de uso (VERSCHUEREN, 2016). Daí a importância de trabalhos que contemplem uma maior variedade de idiomas. Como veremos mais adiante, apesar de existirem diversos estudos que se inserem na perspectiva da Pragmática cross-cultural, poucos deles contemplam mais que duas línguas e, quando o fazem, costumam dar ênfase àquelas amplamente estudadas desse ponto de vista, como o inglês.

Para preencher essa lacuna, o Grupo de Pesquisa em Pragmática (inter)linguística, cross-cultural e intercultural (GPP) ${ }^{3}$ está realizando projetos, que levam em conta essas reflexões. O trabalho apresentado aqui, que se insere no filão dos estudos cross-culturais, focaliza-se, em especial, na realização do ato de fala do pedido e busca comparar quatro línguas: italiano, português brasileiro, espanhol argentino e alemão. Os dados foram coletados por meio de um Discourse Completion Task escrito (DCT) e a análise concentrou-se na comparação das características do ato principal (head act), isto é, o núcleo da sequência por meio da qual se realiza um pedido e em que é veiculada a força ilocucionária (BLUM-KULKA et al., 1989, p. 275). O objetivo foi identificar as características do pedido nas línguas e culturas escolhidas, verificando, sobretudo, a existência de convergências, divergências e

3 O grupo, cadastrado desde 2013, no Diretório de Grupos de Pesquisa do CNPq (http://dgp.cnpq.br/dgp/espelhogrupo/36583\#identificacao), desenvolve pesquisas sobre atos de fala e fenômenos pragmáticos em diferentes línguas e perspectivas. Para mais informações sobre o grupo: https://www.gppragmatica-usp.com/. 


\section{PERcursos Linguísticos • Vitória (ES) •v. $10 \bullet$ •n. $26 \bullet 2020 \bullet$ ISSN: 2236-2592 • Dossiê: Linguagem, Comunicação e Cognição •}

recorrências que possam revelar a relação entre suas estruturas significantes e as operações de conceitualização realizadas pelos falantes.

\section{Língua, cultura e cognição: o pedido como indício de esquemas conceituais}

Língua, cultura e cognição são categorias fortemente correlacionadas: não são entidades abstratas, mas modelos nos quais se baseiam o comportamento, o discurso e o modo de pensar de uma determinada comunidade de fala. Na perspectiva adotada para a realização deste estudo, consideramos que a língua não é, portanto, apenas um código composto por estruturas gramaticais e formas lexicais, mas o produto da atividade cognitiva humana. Além disso, partimos do pressuposto de que as estruturas linguísticas significantes estão sempre relacionadas às operações de conceitualização dos falantes, pois existe uma passagem do plano cognitivo para a verbalização, que revela esquemas conceituais e culturais.

Em outras palavras, quando falamos, estamos sempre não apenas produzindo enunciados, mas também transformando em materialidade linguística nossa visão de mundo e as categorias da cultura e da sociedade às quais pertencemos, que criam uma convencionalidade por meio da qual nossas falas podem ser reconhecidas e interpretadas.

Acontece, assim, que os esquemas conceituais, que derivam tanto da nossa percepção de mundo quanto das referências culturais e experienciais, atualizam-se em esquemas linguísticos. Na visão de Goddard e Wierzbicka (2004), se trataria de verdadeiros "scripts", isto é, de normas culturalmente específicas na comunicação, que regulam a maneira de pensar, de se expressar e de se comportar, tendo como consequência diferenciações também na realização dos atos de fala e mudando, além disso, a maneira como são percebidos outros elementos essenciais na comunicação como o contexto, considerado não apenas "dado", mas sempre também "construído" pelos participantes de uma interação (SBISÀ, 2002), justamente a partir das referências de cada falante e da comunidade à qual pertence. Resumindo, poderíamos afirmar que os indivíduos, ao falar, estão sempre se referindo a categorias linguísticas, 


\section{PERcursos Linguísticos • Vitória (ES) •v. 10 •n. 26・2020 • ISSN: 2236-2592 • Dossiê: Linguagem, Comunicação e Cognição •}

por sua vez, relacionadas a categorias conceituais, que podem revelar características peculiares e traços distintivos da(s) língua(s) e da(s) cultura(s).

Uma das formas pelas quais se manifestam essas categorias conceituais é o modo como aparecem linguisticamente o "eu" e o "outro" e, portanto, a ideia de subjetividade e intersubjetividade. Nesse sentido, podemos observar as línguas, classificando os atos de fala, por exemplo, de acordo com a perspectiva adotada no seu proferimento, que pode evidenciar a quem se atribui a responsabilidade pela realização do ato e indica se e em que medida o falante se sente autorizado a "invadir" o território do outro, oferecendo, dessa maneira, possíveis indícios sobre a representação que o falante tem de si mesmo e de seu interlocutor. Como lemos em Blum-Kulka et al., "[the] choice of perspective affects social meaning" (1989, p. 19), por isso, evitar envolver o interlocutor pode reduzir o nível de coercividade. Isso torna-se especialmente relevante em atos de fala como o pedido, que são potencialmente ameaçadores à face (Face Threatening Acts - FTAs, na terminologia de Brown e Levinson, 1987).

O que muda, então, se os falantes de uma determinada língua escolhem com maior frequência colocar o foco em si mesmos ou no(s) interlocutor(es)? E se o ato de fala for orientado pela primeira do plural (nós)? Que indícios sobre o possível pertencimento do falante e do ouvinte ao mesmo grupo isso pode evidenciar? E se, ainda, a perspectiva adotada for impessoal, afastando a responsabilidade pelo ato tanto do falante quanto do ouvinte?

Outro aspecto que pode fornecer indícios significativos sobre tendências no modo como se configura a relação entre o "eu" e o "outro" é o grau de diretividade ou indiretividade dos atos de fala. Como afirma Searle:

In indirect speech acts the speaker communicates to the hearer more than he actually says by way of relying on their mutually shared background information, both linguistic and nonlinguistic, together with the general powers of rationality and inference on the part of the hearer (1975, p. 169).

Com efeito, um ato de fala indireto exige um maior esforço de inferência por parte do outro e, consequentemente, pressupõe a existência de conhecimentos compartilhados e da solidariedade do interlocutor, partes essenciais do conhecido 


\section{PERcursos Linguísticos • Vitória (ES) •v. $10 \bullet n .26 \bullet 2020 ・$ ISSN: 2236-2592 • Dossiê: Linguagem, Comunicação e Cognição •}

Princípio de Cooperação (GRICE, 1975), ao mesmo tempo em que pressupõe a tentativa de não invadir demasiadamente seu espaço, demonstrando assim respeito por sua liberdade e por seu "território". Por outro lado, com um ato de fala direto, diminui a necessidade de inferência do interlocutor e, paralelamente, o falante demonstra uma menor preocupação com a invasão do espaço do outro.

É o que acontece quando, no caso dos pedidos, o falante pode escolher entre um ato direto como "me dá um copo d’água" e outro que, no grau máximo de indiretividade, se limita a uma afirmação como "estou com sede", exigindo a "cooperação" do interlocutor.

Nesse equilíbrio entre as diferentes posturas que o falante pode assumir, outro fator que desenvolve um papel importante é o da convencionalidade: quanto menos convencional a indiretividade, maior o esforço exigido de quem participa da interação. É por isso que as pesquisas como a de Blum-Kulka et al. (1989) mostram que, com frequência, a estratégia preferida são atos convencionalmente indiretos, ou seja, atos que utilizam fórmulas indiretas mas "codificadas" e, por isso, facilmente reconhecíveis. Ainda pensando nos pedidos, um exemplo como "você poderia me dar um copo d'água?" se colocaria nessa posição intermediária. Assim, o falante se mostra preocupado com a invasão do território do outro, mas, ao mesmo tempo, não corre o risco de o interlocutor não entender o valor ilocucionário de seu ato.

\section{Pedidos e outros atos de fala em perspectiva cross-cultural: alguns estudos}

Existem diversos estudos cross-culturais que lançam mão dos DCTs para entender como são realizados atos de fala em diferentes línguas, como os que comparam pedidos de desculpa em inglês e polonês (SUSZCZYŃSKA, 1999) ou em inglês e árabe (BATAINEH; BATAINEH, 2008). Há também trabalhos que analisam pedidos em francês e holandês (VAN MULKEN, 1996) e em inglês americano e japonês (HILL; IDE; IKUTA; KAWASAKI; OGINO, 1986), enquanto outros confrontam as recusas em inglês americano com aquelas realizadas em coreano (KWON, 2004) ou em espanhol mexicano (FÉLIX-BRASDEFER, 2008). Como vemos, a maioria desses trabalhos tende a se concentrar na comparação entre apenas 


\section{PERcursos Linguísticos • Vitória (ES) •v. 10 •n. 26 • 2020 • ISSN: 2236-2592 • Dossiê: Linguagem, Comunicação e Cognição •}

duas línguas, uma das quais é frequentemente o inglês, incorrendo, portanto, por um lado, na dificuldade de identificar padrões (cf. VERSCHUEREN, 2016) e, por outro, no reforço da hegemonia de algumas línguas em detrimentos de outras, com a consequente limitação do alcance dos resultados. A seguir, descreveremos com maiores detalhes os estudos que, como o do GPP, foram concebidos com o objetivo de comparar uma variedade maior de línguas: o trabalho seminal de Blum-Kulka et al. (1989), a pesquisa de Ogiermann sobre pedidos (2009) e o estudo mais recente de Nuzzo e Cortés-Velásquez (2020).

Em primeiro lugar, destacamos o Cross-Cultural Speech Act Realization Project (CCSARP), realizado por Blum-Kulka, House e Kasper e publicado em 1989. Esse trabalho se concentra na comparação, em sete línguas e/ou variedades (inglês americano, britânico e australiano, alemão, francês canadense, dinamarquês e hebraico), de pedidos e pedidos de desculpas, dois FTAs (BROWN; LEVINSON, 1987). O projeto tinha o objetivo de investigar a variação cross-cultural, ou seja, diferenças e semelhanças na realização dos atos de fala nas línguas envolvidas, assim como a variação sociopragmática. Para fazer isso, as autoras criaram um DCT em que foram manipuladas as variáveis de poder e distância social, de forma a contemplar contextos variados. Os resultados apontam semelhanças e diferenças cross-culturais: por um lado, as variáveis contextuais influenciaram a realização dos atos de fala, com algumas situações favorecendo o uso de estratégias similares em todas as línguas; por outro lado, as realizações dos atos demonstram que há diferenças cross-culturais no estilo interacional, principalmente no que tange ao grau de (in)diretividade.

Diversos outros estudos cross-culturais se inspiraram metodologicamente no trabalho e nos métodos de Blum-Kulka et al. (1989). Entre eles, por exemplo, o estudo de Ogiermann (2009), concentrado em pedidos, reclamações e pedidos de desculpas, realizados em inglês britânico, alemão, polonês e russo. Ao analisar a diretividade dos pedidos, a autora percebeu que os britânicos e os alemães foram mais indiretos, ao passo que os poloneses e os russos foram mais diretos, utilizando com frequência o imperativo. A análise da perspectiva também mostrou resultados interessantes: britânicos e alemães realizaram seus pedidos principalmente na primeira pessoa (perspectiva do 'eu'), ao passo que russos e poloneses deram preferência para a 


\section{PERcursos Linguísticos • Vitória (ES) •v. $10 \bullet$ •n. $26 \bullet 2020 \bullet$ ISSN: 2236-2592 • Dossiê: Linguagem, Comunicação e Cognição •}

segunda pessoa (perspectiva do 'tu'). O uso de pedidos mais diretos, com o imperativo, e o reconhecimento do papel do ouvinte na realização do pedido por meio da perspectiva do 'tu' levaram a autora a concluir que os pedidos nas situações contempladas são vistos como menos ameaçadores à face negativa nas culturas russa e polonesa em comparação com a britânica e a alemã.

Entre os estudos mais recentes, lembramos aqui o trabalho de Nuzzo e Cortés Velásquez (2020) sobre outro FTA: desmarcar compromissos de última hora (last minute cancellations). O projeto contemplou na primeira fase italiano e espanhol colombiano, mas, posteriormente, foram incluídas outras línguas: francês, mandarim, inglês britânico e estadunidense, português peninsular, espanhol peninsular e mexicano e ucraniano. Por enquanto, porém, foram publicados somente os dados que comparam as respostas de falantes italianos e colombianos. As respostas foram analisadas por meio de uma metodologia em parte baseada no CCSARP e em parte criada a partir dos próprios dados analisados (data-driven), levando a distinguir semelhanças e diferenças entre os dois grupos de informantes. Uma diferença encontrada diz respeito às estratégias de cortesia: os italianos preferem as de cortesia negativa, no intuito de reduzir a invasão no território do interlocutor, ao passo que os colombianos lançam mão daquelas de cortesia positiva, estabelecendo um vínculo maior com o interlocutor e apelando para sua solidariedade.

\section{Coleta e análise dos dados: escolhas metodológicas}

Nesta seção, trataremos da metodologia escolhida para esta pesquisa de caráter exploratório: apresentaremos o instrumento da coleta de dados, descreveremos a taxonomia utilizada para a análise dos pedidos e traçaremos o perfil dos informantes, que forneceram os dados analisados.

\section{- A coleta de dados por meio de DCTs}




\section{PERcursos Linguísticos • Vitória (ES) •v. 10 •n. 26 • 2020 • ISSN: 2236-2592 • Dossiê: Linguagem, Comunicação e Cognição •}

O instrumento utilizado para a coleta dos dados foi, como nos estudos mencionados, o DCT, um questionário ao qual o falante responde, escrevendo o que ele diria nas situações propostas.

O DCT recebeu críticas por apresentar algumas limitações, entre as quais, a falta de interação, que não permite a co-construção dos atos de fala (KERBRATORECCHIONI, 2005); o uso da escrita para representação a oralidade; o distanciamento da conversa espontânea, pois, ao preencher o questionário, o informante escreve o que ele pensa que seria adequado dizer e não necessariamente o que de fato diria na situação descrita (KASPER, 2008).

Por outro lado, o DCT apresenta importantes vantagens para estudos crossculturais como o que nos propomos a conduzir. Em primeiro lugar, é um instrumento que pode ser traduzido para outras línguas e distribuído com relativa facilidade, especialmente porque pode ser amplamente divulgado pela internet, tornando possível a coleta de um grande número de dados comparáveis (BLUM-KULKA et al., 1989; BILLMEYER; VARGHESE, 2000). Em segundo lugar, porque o DCT, diferentemente de dados naturalísticos, permite manipular as variáveis contextuais, como a distância social, o grau de imposição e o setting (BROWN; LEVINSON, 1987; SWEENEY; ZHU, 2016; OGIERMANN, 2018). Por fim, justamente pelo fato de os informantes escreverem o que eles acham que seria adequado dizer, as respostas são particularmente úteis para ter acesso a esquemas e referências culturais dos informantes (BLUM-KULKA et al., 1989; FÉLIX-BRASDEFER, 2010; NUZZO; GAUCI, 2014; OGIERMANN, 2018).

Além disso, por meio dos DCTs, é possível conhecer diferentes perspectivas que ajudam a entender os efeitos pragmáticos. Kádar \& Haugh (2013) propuseram uma subdivisão em duas categorias: (a) na primeira, dita first order, é o falante quem fornece pistas e indícios, que vão permitir acessar não apenas a sua maneira de dizer, mas também, paralelamente, a de perceber, sentir e entender o mundo; considera-se, inclusive, a possibilidade de o falante indicar sua avaliação em uma interação da qual participou, o que implica uma conceitualização do fenômeno tanto interna ou émica, quanto externa ou ética; (b) na segunda, chamada second order, as pistas para a explicação dos fenômenos em análise são dadas pelo pesquisador, mas também por 


\section{PERcursos Linguísticos • Vitória (ES) •v. 10 •n. 26・2020 • ISSN: 2236-2592 • \\ Dossiê: Linguagem, Comunicação e Cognição •}

observadores leigos ${ }^{4}$, que permitem ver o objeto de estudo a partir de diferentes perspectivas, enriquecendo uma possível análise, para a qual o pesquisador buscará a melhor metalinguagem (Quadro 1).

Quadro 1 - First order e second order para entender fenômenos pragmáticos

\begin{tabular}{|l|l|}
\hline \multicolumn{1}{|c|}{ First order } & \multicolumn{1}{c|}{ Second order } \\
\hline $\begin{array}{l}\text { (1) Compreensão do } \\
\text { participante/metaparticipante }\end{array}$ & $\begin{array}{l}\text { (3) Compreensão do analista e do } \\
\text { observador leigo }\end{array}$ \\
\hline $\begin{array}{l}\text { (2) Conceptualização émica (interna) e } \\
\text { ética (externa) }\end{array}$ & $\begin{array}{l}\text { (4) Conceitualização teórica (baseada } \\
\text { também na percepção comum) }\end{array}$ \\
\hline
\end{tabular}

Fonte: As autoras (2020).

O DCT utilizado neste trabalho inclui oito situações diferentes, em que são eliciados pedidos, com a manipulação das variáveis grau de imposição e distância social, além dos settings (público/privado) (BROWN; LEVINSON, 1987; NICKELS, 2006), para que fossem representadas todas as possíveis combinações (SANTORO, $2017)^{5}$.

\section{- Analisar os atos principais: uma taxonomia}

A taxonomia utilizada para a análise dos pedidos coletados por meio do DCT descrito acima é inspirada no trabalho de Blum-Kulka et al. (1989), mas foi adaptada pelo GPP a partir dos dados (data driven), visando a atender às especificidades dos corpora analisados. Para cada pedido, em primeiro lugar, foram isolados os atos

\footnotetext{
${ }^{4}$ Para um exemplo de estudo realizado com a participação de observadores não especialistas, se veja SILVA NETO; SANTORO, 2015.

${ }^{5}$ Para maiores informações sobre as situações do questionário, remetemos a sua versão online: https://forms.gle/egb2AYnSwc3NKBBG6
} 


\section{PERcursos Linguísticos • Vitória (ES) •v. 10 •n. 26・2020 • ISSN: 2236-2592 • Dossiê: Linguagem, Comunicação e Cognição •}

principais, posteriormente classificados, com base no grau de (in)diretividade em quatro categorias (Quadro 2):

a. atos diretos (ap1), nos casos em que o pedido é realizado por meio de imperativos (dá água aî), verbos performativos (queria pedir um copo d'água), want-statements (queria um copo d'água) e perguntas diretas (você me dá um copo d'água?);

b. atos convencionalmente indiretos (ap2), realizados por meio de propostas, expressões de necessidade (preciso de um copo d'água), verificação de condições, frequentemente por meio do modal "poder" (você poderia me dar um copo d'água?);

c. atos não convencionalmente indiretos (ap3), em que o pedido não é mencionado, mas o falante fornece pistas fortes ou fracas, a serem “completadas" pelo interlocutor (estou com sede);

d. ato principal inexistente (ap0), nos casos em que não há um ato principal com a força ilocucionária de pedido.

Em seguida, para cada ato principal, identificou-se a perspectiva utilizada: a perspectiva do 'eu' (pp1), a do ‘tu' (pp2), a do 'nós' (pp3) e a impessoal (pp4) (Quadro 2), de acordo com o que foi explicado anteriormente.

Quadro 2: Classificação dos atos principais: (in)diretividade e perspectiva

\begin{tabular}{|l|l|l|}
\hline Grau de (in)diretividade & ap0 & Inexistente \\
\cline { 2 - 3 } & ap1 & Direto \\
\cline { 2 - 3 } & ap2 & Convencionalmente indireto \\
\cline { 2 - 3 } & ap3 & Não convencionalmente indireto \\
\hline Perspectivas & pp1 & 1a pessoa singular (eu) \\
\hline
\end{tabular}


Dossiê: Linguagem, Comunicação e Cognição •

\begin{tabular}{|l|l|l|}
\hline & $\mathrm{pp} 2$ & 2a pessoa singular ou plural (tu/você(s)) \\
\cline { 2 - 3 } & $\mathrm{pp} 3$ & 1a pessoa plural (nós) \\
\cline { 2 - 3 } & $\mathrm{pp} 4$ & Impessoal \\
\hline
\end{tabular}

Fonte: As autoras (2020).

A classificação dos atos de fala foi realizada por meio de um sistema de etiquetas, que tornou os corpora consultáveis por meio do software AntConc (ANTHONY, 2011), usado na Linguística de Corpus.

\section{- Os informantes}

As análises descritas acima foram conduzidas em corpora constituídos pelas respostas de uma parte de todos os informantes que responderam ao DCT. Inicialmente, para garantir uma seleção homogênea, decidiu-se analisar as respostas de 30 informantes nativos de cada língua, com exceção do alemão, pois, no momento das análises, só haviam respondido 18 informantes dessa língua (Quadro 3).

Quadro 3 - Os informantes

\begin{tabular}{|c|c|c|c|c|c|}
\hline & & $\begin{array}{l}\text { Português } \\
\text { brasileiro }\end{array}$ & Italiano & $\begin{array}{l}\text { Espanhol } \\
\text { argentino }\end{array}$ & Alemão \\
\hline \multicolumn{2}{|c|}{ Número de informantes } & 30 & 30 & 30 & 18 \\
\hline \multicolumn{2}{|c|}{ Procedência } & $\begin{array}{c}\text { de São Paulo } \\
\text { e arredores }\end{array}$ & $\begin{array}{c}10 \text { do Norte } \\
10 \text { do Centro } \\
10 \text { do Sul }\end{array}$ & $\begin{array}{l}\text { Majoritaria- } \\
\text { mente de } \\
\text { Córdoba }\end{array}$ & $\begin{array}{l}\text { Majoritaria- } \\
\text { mente de } \\
\text { Potsdam }\end{array}$ \\
\hline \multirow[t]{3}{*}{ Idade } & $18-30$ & 14 & 12 & 19 & 12 \\
\hline & $31-40$ & 5 & 4 & 3 & 3 \\
\hline & $41-50$ & 6 & 7 & 2 & 0 \\
\hline
\end{tabular}


PERcursos Linguísticos • Vitória (ES) •v. 10 •n. 26 • 2020 • ISSN: 2236-2592 •

Dossiê: Linguagem, Comunicação e Cognição •

\begin{tabular}{|l|c|c|c|c|c|}
\hline & $>\mathbf{5 1}$ & 5 & 7 & 6 & 3 \\
\hline Gênero & $\mathbf{F}$ & 19 & 22 & 22 & 13 \\
\cline { 2 - 6 } & $\mathbf{M}$ & 11 & 8 & 8 & 5 \\
\hline
\end{tabular}

Fonte: As autoras (2020).

Quanto à distribuição geográfica, no caso da Itália, foram contemplados informantes provenientes do norte, do centro e do sul do país, nas mesmas proporções. No caso das outras línguas, no entanto, a maioria dos informantes tinha origem na mesma cidade: os informantes brasileiros são da grande São Paulo, os argentinos, da região de Córdoba e os alemães, da cidade de Potsdam. Considerando a distribuição de outras variáveis sociolinguísticas, como a idade e o gênero, há semelhanças entre os quatro grupos: a maioria (cerca de 50\%) dos informantes têm entre 18 e 30 anos e dois terços deles são do sexo feminino.

\section{(In)diretividade e perspectivas nos pedidos: resultados e discussão}

A partir da anotação manual dos corpora, realizada selecionando etiquetas para cada tipo de ato principal e perspectiva, foi possível obter os resultados que passamos agora a descrever e discutir.

Iniciando pelos resultados gerais relativos aos atos principais, vemos no gráfico abaixo (Gráfico 1) que, mesmo tendo sido realizada com línguas distintas, nossa pesquisa evidencia tendências similares às apontadas pelo estudo de Blum-Kulka et al. (1989), resumidas a seguir: os atos convencionalmente indiretos (ap2) constituem a estratégia mais utilizada pelos falantes de todas as línguas, embora na comparação entre argentinos $(58,8 \%)$ e brasileiros $(69,6 \%)$ seja possível perceber uma diferença de mais de 10 pontos percentuais. De fato, os argentinos tendem a utilizar atos diretos (ap1) com uma frequência maior $(27,9 \%)$ em relação aos italianos $(18,7 \%)$ e mais ainda se comparados com os brasileiros (14,2\%). Os dados de argentinos e alemães mostram, nesse aspecto, uma diferença mais expressiva, já que os primeiros escolhem 
atos diretos (ap1) 27,9\% das vezes, enquanto os segundos em apenas 1,4\% dos casos. Chama a atenção, além disso, a presença de atos principais não convencionalmente indiretos (ap3) no alemão, cuja porcentagem $(32,7 \%)$ chega a ser quase três vezes superior em relação às outras três línguas que, ao contrário, se mantêm no mesmo patamar (entre $11,2 \%$ e $12,9 \%$ ).

Mesmo que com uma presença consideravelmente menor, outro aspecto que se sobressai diz respeito às porcentagens dos atos principais inexistentes (ap0), que ficam abaixo de $1 \%$ no caso de alemão e espanhol, chegam a $2 \%$ no italiano, e a $5 \%$ no português brasileiro, evidenciando, neste último caso, uma tendência mais marcada ao evitamento do pedido.

Gráfico 1 - A distribuição dos atos principais dos pedidos nas quatro línguas

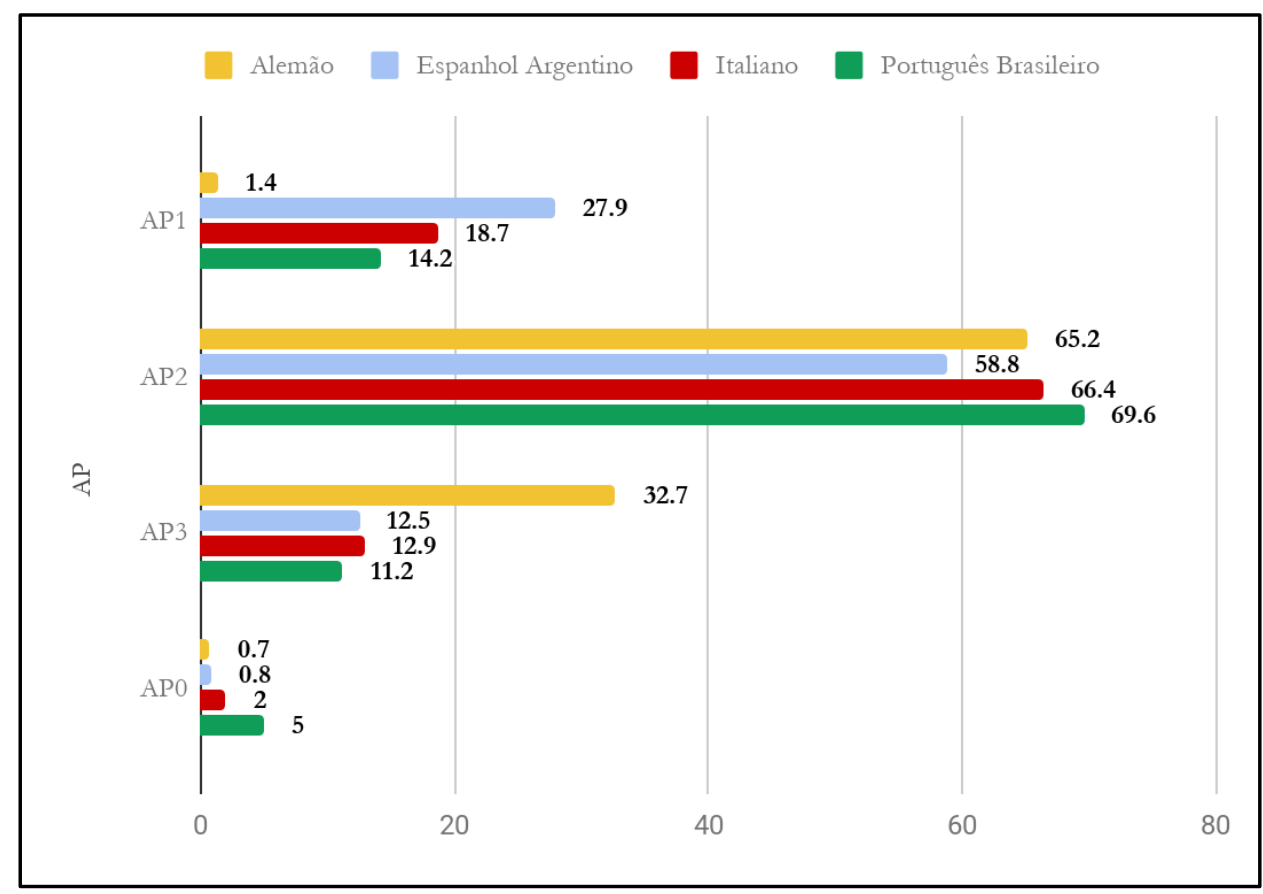

Fonte: As autoras (2020).

Retomando e buscando esquematizar o que já escrevemos no início deste texto a respeito de processos cognitivos, categorias e representações que o grau de (in)diretividade pode indicar, o quadro a seguir (Quadro 4) mostra que, na nossa interpretação, a necessidade de inferência se torna maior com o aumento da 


\section{PERcursos Linguísticos • Vitória (ES) •v. 10 •n. 26 • 2020 • ISSN: 2236-2592 • Dossiê: Linguagem, Comunicação e Cognição •}

indiretividade, até chegar ao ato de fala inexistente (ap0), o qual pode ser escolhido por, ao menos, duas razões:

(a) pelo fato de não ser considerado aceitável, em determinadas culturas, realizar alguns tipos de pedido;

(b) pela escolha de deixar ao interlocutor o papel de "completar" o que não foi dito, que caracterizaria as sociedades denominadas "high-context" por Hall (1976), isto é, aquelas em que o contexto possui um papel dominante e espera-se a participação do outro.

Nos nossos dados, os brasileiros evitam a realização do pedido com uma frequência superior em relação às outras línguas, o que poderia indicar uma presença mais marcada desse traço. Do lado oposto, nos dados dos argentinos, evidencia-se a maior presença de atos diretos (ap1), o que apontaria para uma sociedade que na terminologia de Hall (1976) seria chamada "low-context". Como se vê no quadro a seguir, é preciso, além disso, levar em conta que uma maior necessidade de inferência é diretamente proporcional à confiança na solidariedade do outro e inversamente proporcional à invasão do espaço alheio. Observando mais uma vez os dados, é possível, portanto, identificar uma tendência mais acentuada dos argentinos nesse sentido, para os quais, todavia, também o fator do pertencimento ao grupo parece desempenhar um papel relevante. No outro extremo, os dados dos alemães evidenciam uma presença muito maior que nas outras línguas de atos não convencionalmente indiretos (ap3), o que implica pressupor um grau elevado de inferência e confiança na solidariedade do outro, e, paralelamente, uma baixa invasão do espaço alheio com a consequente preservação do território.

Todavia, a predominância em todas as línguas de atos convencionalmente indiretos (ap2) confirma que as estratégias preferidas são as intermediárias, percebidas pela maioria como mais "seguras", pois garantem que o pedido seja compreendido, sem, no entanto, exigir demais do interlocutor ou invadir seu espaço.

Quadro 4 - Grau de (in)diretividade, categorias e representações 
PERcursos Linguísticos • Vitória (ES) •v. 10 •n. 26 • 2020 • ISSN: 2236-2592 •

Dossiê: Linguagem, Comunicação e Cognição •

\begin{tabular}{|l|l|l|l|}
\hline & Inferência & $\begin{array}{l}\text { Confiança na solidariedade do } \\
\text { outro }\end{array}$ & $\begin{array}{l}\text { "Invasão" do espaço do } \\
\text { outro/pertencimento ao } \\
\text { grupo }\end{array}$ \\
\hline ap0 & +++ & +++ & - \\
\hline ap3 & ++ & ++ & + \\
\hline ap2 & + & + & ++ \\
\hline ap1 & - & - & +++ \\
\hline
\end{tabular}

Fonte: As autoras (2020).

A análise das perspectivas adotadas pelos falantes é outro elemento, por meio do qual podemos constatar a presença de características definidoras de línguas e culturas, delineadas observando a relação que se estabelece entre o "eu" e o "outro". Se, por um lado, há estratégias que tendem a preservar o espaço e a imagem do outro, se mostram também escolhas nas quais ficam evidentes a invasão do território e a ameaça da imagem do interlocutor. As preferências na formulação de pedidos pode evidenciar: (a) a quem se atribui a responsabilidade pela realização - ou não - do ato; (b) se e em que medida o falante se sente autorizado a invadir o espaço do outro e a ameaçar sua imagem negativa, isto é, seu território.

O próximo gráfico (Gráfico 2) contém os resultados obtidos nas quatro línguas focalizando a perspectiva.

Gráfico 2 - A distribuição das perspectivas dos pedidos nas quatro línguas 


\section{PERcursos Linguísticos • Vitória (ES) •v. 10 •n. 26 • 2020 • ISSN: 2236-2592 • \\ Dossiê: Linguagem, Comunicação e Cognição •}

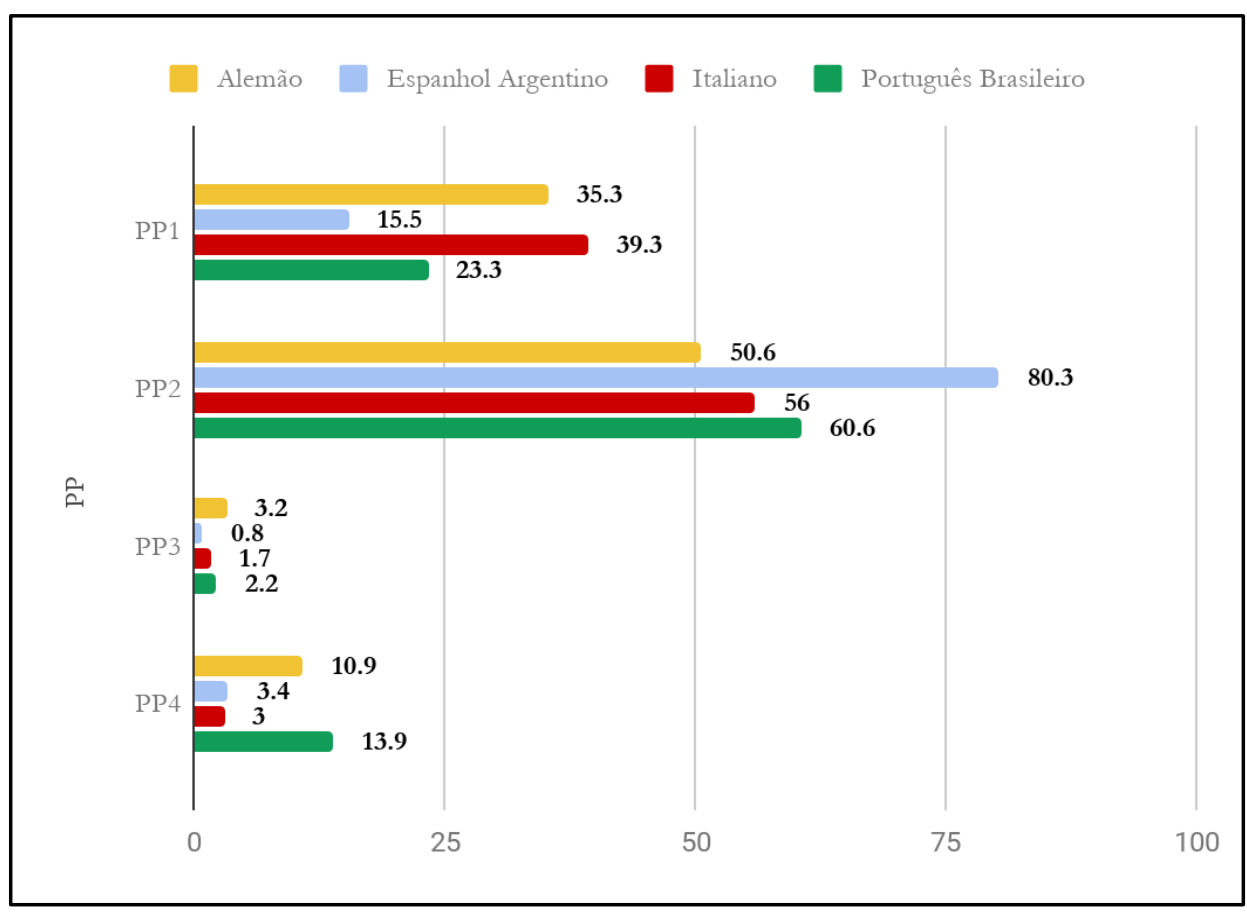

Fonte: As autoras (2020).

Como se vê, a perspectiva de segunda pessoa $(t u)$, isto é, aquela que coloca o interlocutor em primeiro plano, atribuindo a ele a responsabilidade pelo cumprimento do pedido, é a que predomina com porcentagens que, em todas as línguas, superam $50 \%$, chegando a mais de $80 \%$ no caso dos argentinos. Esse dado confirma uma característica evidenciada também pela análise do grau de diretividade, isto é, que a sociedade argentina parece pautada por uma tendência a se sentir autorizado a invadir o espaço do outro, que se revela maior em relação às outras línguas examinadas, provavelmente porque esse traço se relaciona com a importância de enfatizar o pertencimento a um mesmo grupo e a força da proximidade.

De fato, consideramos que a perspectiva do "tu" (pp2) é a que mais responsabiliza o interlocutor e invade seu território (v. Quadro 5), especialmente no caso de um ato diretivo como o pedido, porque desvia o curso de suas ações e o obriga a uma reação, mesmo no caso de não poder ou não querer realizar o que foi solicitado. Por outro lado, embora esse aspecto se preste a uma dupla interpretação, essa escolha pode indicar proximidade e pertencimento ao mesmo grupo, já que o falante acredita 


\section{PERcursos Linguísticos • Vitória (ES) •v. 10 •n. 26・2020 • ISSN: 2236-2592 • \\ Dossiê: Linguagem, Comunicação e Cognição •}

poder "convocar" seu interlocutor.

Quadro 5 - Perspectivas nos pedidos: categorias e representações

\begin{tabular}{|l|l|l|}
\hline & $\begin{array}{l}\text { Responsabilização do } \\
\text { interlocutor pela } \\
\text { realização do ato }\end{array}$ & $\begin{array}{l}\text { "Invasão" do espaço do outro e/ou } \\
\text { pertencimento ao mesmo grupo/ } \\
\text { proximidade }\end{array}$ \\
\hline $\begin{array}{l}\text { pp1 } \\
(\text { eu })\end{array}$ & -- & -+ \\
\hline $\begin{array}{l}\text { pp2 } \\
(t u)\end{array}$ & ++ & +++ \\
\hline $\begin{array}{l}\text { pp3 } \\
(\text { nós })\end{array}$ & +- & $--/++$ \\
\hline $\begin{array}{l}\text { pp4 } \\
\text { (imp) }\end{array}$ & --- & --- \\
\hline
\end{tabular}

Fonte: As autoras (2020).

Observando ainda o gráfico e correlacionando-o com o quadro 5, percebe-se que outro dado interessante diz respeito ao uso da perspectiva em primeira pessoa (pp1). Nesse caso, as línguas se dividem em dois blocos com comportamentos similares: por um lado, espanhol argentino e português brasileiro com porcentagens que, embora não idênticas, se colocam em um nível mais baixo (respectivamente, $15,5 \%$ e $23,3 \%$ ); por outro lado, alemão e italiano que superam em mais de 10 pontos percentuais o bloco das primeiras duas línguas e apresentam um nível bem mais elevado de uso da primeira pessoa (respectivamente, 35,3\% e 39,3\%). Uma primeira possível explicação pode ser encontrada no próprio funcionamento das línguas. Com efeito, se em alemão e italiano pedir em primeira pessoa é comum, em português brasileiro e espanhol argentino não há formas equivalentes para a elaboração de pedidos. Todavia, no momento da análise, cabe lembrar o que se afirma no texto de Blum-Kulka et al. sobre esse aspecto: "Choice of perspective presents an important source of variation in requests; since requests are inherently imposing, avoidance to name the hearer as actor can reduce the form's level of coerciveness". (1989, p. 19) 


\section{PERcursos Linguísticos • Vitória (ES) •v. $10 \bullet n .26 \bullet 2020 ・$ ISSN: 2236-2592 • Dossiê: Linguagem, Comunicação e Cognição •}

Tendo isso em vista, podemos interpretar a escolha da perspectiva do "eu" (pp1) também como uma “deresponsabilização" do interlocutor pela realização do ato (v. Quadro 5), que, colocando o "eu" em primeiro plano, diminui o efeito de coercitividade em relação ao "tu" e à invasão do espaço do outro.

As duas últimas observações se referem à perspectiva do "nós" (pp3) e ao impessoal (pp4). No primeiro caso, a responsabilidade pelo cumprimento da ação solicitada é aparentemente compartilhada entre falante e interlocutor, criando-se, assim, também a ilusão de que ambos pertencem ao mesmo grupo. Em espanhol argentino, essa opção foi escolhida por menos de $1 \%$ dos informantes $(0,8 \%)$, em italiano e português brasileiro ficou por volta de $2 \%$ (respectivamente, 1,7\% e 2,2\%), enquanto em alemão chegou a 3,2\%, com uma porcentagem que, mesmo mantendo-se baixa, indica que a estratégia está entre as opções consideradas pelos falantes.

No que diz respeito ao impessoal, as porcentagens de argentinos e italianos ficaram por volta de $3 \%$, enquanto os alemães chegaram a $10,9 \%$ e os brasileiros a 13,9\%. Essa escolha, por ser a que menos responsabiliza os envolvidos na interação, pode ser interpretada como o desejo do falante de criar um efeito de proteção do espaço do outro. Se no caso dos alemães isso pode remeter à importância, já evidenciada anteriormente, de não comprometer o outro, evitando de entrar no território alheio, quando se trata dos brasileiros poderia, verossimilmente, ser interpretada como uma estratégia para evitar também comprometer a si mesmo, em direção ao evitamento, como foi evidenciado no caso da não realização do ato (ap0).

\section{Conclusão e perspectivas futuras}

Vimos neste artigo que a análise linguística dos pedidos na perspectiva da Pragmática cross-cultural permite reconhecer esquemas e referências ligados às culturas, de modo que as diferenças na realização dos pedidos em português brasileiro, italiano, espanhol argentino e alemão podem revelar categorias conceituais e esquemas culturais de referência, que se manifestam na(s) língua(s).

Também no caso em que há analogias do ponto de vista quantitativo, as 


\section{PERcursos Linguísticos • Vitória (ES) •v. 10 •n. 26 • 2020 • ISSN: 2236-2592 • Dossiê: Linguagem, Comunicação e Cognição •}

interpretações e as categorias conceituais de referência não são necessariamente as mesmas e as análises precisam levar em conta o conjunto dos resultados.

Em geral, podemos dizer que, observando os atos de fala principais e as perspectivas nos pedidos produzidos pelos falantes das quatro línguas escolhidas, evidenciou-se a tendência comum a preferir a indiretividade e a convencionalidade, adotando a estratégia mais segura e limitando a impositividade que o pedido carrega. No que concerne às perspectivas, a escolha indubitavelmente mais frequente envolve no pedido o interlocutor, o que pode ser interpretado como uma tendência nas quatro línguas a considerar aceitável que o "tu” seja incluído no jogo interacional criado para a realização do pedido.

Como observamos, há interessantes diferenças nas análises que comparam as línguas e as culturas e, com elas, indícios de traços e categorias que merecem aprofundamentos futuros para confirmar ou desmentir hipóteses e intuições. Em especial, para refinar as interpretações dos fenômenos linguístico-pragmáticos identificados e observados, abordagens tanto da "first order" quanto da "second order", citadas por Kádar e Haugh (2013, p. 3), poderão contribuir nos próximos estudos: entrevistas retrospectivas com grupos de respondentes, além da avaliação e justificativas das respostas por parte dos falantes poderão certamente acrescentar elementos novos.

Além disso, aumentando o número de informantes e comparando os resultados obtidos por meio dos DCTs escritos com aqueles coletados com outras metodologias como role plays, DCTs orais ou outras interações mais livres será possível ampliar a reflexão e contribuir para a interpretação.

Lembramos, por fim, que, na realização de um FTA, um pedido no nosso caso, é verdade que as escolhas linguísticas precisam satisfazer uma necessidade concreta do falante para que ele obtenha o que deseja, mas é também verdade que, ao mesmo tempo, existe uma necessidade identitária (CAFFI, 2009) e um desejo de cada indivíduo de "ser visto como quer ser visto". As escolhas são, portanto, determinadas pela junção de, ao menos, esses dois fatores, e influenciadas pelo contexto sociocultural de cada falante e por normas culturais, percepções e avaliações, que 
determinam o modo como falamos e como, pela linguagem, nos relacionamos com o mundo.

\section{Referências}

ANTHONY, L. AntConc (Version 3.5.2) [Computer Software]. Tokyo, Japão: Waseda University, 2017. Disponível em <https://www.laurenceanthony.net/software/antconc/>. Acesso em: 27 out. 2020.

BATAINEH, R. F.; BATAINEH, R. F. Apology strategies of Jordanian EFL university students. Journal of Pragmatics, v. 38, n. 11, p. 1901-1927, 2006.

BILLMYER, K.; VARGHESE, M. Investigating instrument-based pragmatic variability: effects of enhancing discourse completion tests. Applied Linguistics, v. 21, n. 4, p. 517-552, dez. 2000.

BLUM-KUlKA, S.; HOUSE, J. J.; KASPER, G. Cross-Cultural Pragmatics: Requests and Apologies. Norwood, New Jersey: Alex Publishing Corporation, 1989. 300 p.

BROWN, P.; LEVINSON, S. Politeness: Some Universals in Language Use. 2a ed. Cambridge: Cambridge University Press, 1987. 345 p.

CAFFI, C. Pragmatica sei lezioni. 1a ed., Roma: Carocci editore, 2009. 164 p.

FELIX-BRASDEFER, J. C. Politeness in Mexico and the United States: a contrastive study of the realization and perception of refusals. Amsterdam; Philadelphia: John Benjamins, 2008. 195 p.

Data collection methods in speech act performance: DCTs, role plays, and verbal reports. In: MARTÍNEZ FLOR, A.; USÓ-JUAN, E. (Org.). Speech Act Performance. Amsterdam; Philadelphia: John Benjamins, 2010. p.41-56

GODDARD, C.; WIERZBICKA, A. Cultural Scripts: What are they or what are they good for. Intercultural Pragmatics, 1-2, p. 153-166, 2004. 
GRICE, H. P. Logic and Conversation. In: P. Cole (Org.); Speech acts, Syntax and semantics. 1a ed., New York: Academic Press, 1975. p.41-58.

HALL, E. T. Beyond culture. 1a ed. New York: Anchor Books, 1976.

HILL, B.; IDE, S.; IKUTA, S.; KAWASAKI, A.; OGINO, T. Universals of linguistic politeness. Journal of Pragmatics, v. 10, n. 3, p. 347-371, 1986.

KÁDAR, D. Z.; HAUGH, M. Understanding Politeness. Cambridge: Cambridge University Press, 2013. 295 p.

KASPER, G. Data collection in pragmatics. In: SPENCER-OATEY, H. (Org.). Culturally speaking. 2a edição ed. New York: Continuum, 2008, p. 316-341.

KERBRAT-ORECCHIONI, C. Os Atos de linguagem no discurso: teoria e funcionamento. Tradução: Fernando A. De Almeida; Irene E. Dias. 1a ed. Niterói: Editora da Universidade Fluminense, 2005. 220 p.

KWON, J. Expressing refusals in Korean and in American English. Multilingua Journal of Cross-Cultural and Interlanguage Communication, v. 23, n. 4, p. 339364, 2004.

LEECH, G. N. Principles of Pragmatics. London; New York: Longman, 1983. 250 p.

NICKELS, E. L. Interlanguage pragmatics and the effects of setting. Pragmatics and Language Learning, v. 11, p. 253-276, 2006.

NUZZO, Elena; CORTÉS VELÁSQUEZ, D. Canceling Last Minute in Italian and Colombian Spanish: A Cross-Cultural Account of Pragmalinguistic Strategies. Corpus Pragmatics, v. 4, n. 3, p. 333-358, Set. 2020. Disponível em <https://doi.org/10.1007/s41701-020-00084-y>. Acesso em: 27 out. 2020.

OGIERMANN, E. Politeness and in-directness across cultures: A comparison of English, German, Polish and Russian requests. Journal of Politeness Research. Language, Behaviour, Culture, v. 5, n. 2, jan. 2009. Disponível em: <https://www.degruyter.com/doi/10.1515/JPLR.2009.011>. Acesso em: 27 out. 2020.

. Discourse completion tasks. In: JUCKER, A. H.; SCHNEIDER, K. P.; BUBLITZ, W. (Org.). Methods in Pragmatics. Berlin, Boston: De Gruyter, 2018, p. 229-256. 
SANTORO, E. Richieste e attenuazione: un confronto tra italiano e portoghese brasiliano. Normas, v. 7, n. 2, p. 179, 20 dez. 2017. Disponível em: <https://ojs.uv.es/index.php/normas/article/view/11173>. Acesso em: 27 out. 2020.

SAPIR, E. Selected writings of Edward Sapir in language, culture and personality . Berkeley: University of California Press, 1949.

SBISÀ, M. Speech acts in context. Language \& Communication, v. 22, n. 4, p. 421436, out. 2002.

SEARLE, J. Speech Acts. Reprint ed. Oxford: Cambridge University Press, 1969. 208 p.

SILVA NETO, M.; SANTORO, E. A cortesia em pedidos em italiano: um estudo comparativo da percepção de brasileiros e italianos. Revista de Italianística, v. 30, n. 1, p. 80-110, 2015. Disponível em < http://www.revistas.usp.br/italianistica/article/view/116201/113840>. Acesso em: 27 out. 2020.

SUSZCZYŃSKA, M. Apologizing in English, Polish and Hungarian: Different languages, different strategies. Journal of Pragmatics, v. 31, p. 1053-1065, 1999.

SWEENEY, E.; ZHU, H. Discourse Completion Tasks. In: ZHU, H. (Org.). Research Methods in Intercultural Communication: A Practical Guide. Oxford: Wiley Blackwell, 2016, p. 212-222.

VAN MULKEN, M. Politeness markers in French and Dutch requests. Language Sciences, v. 18, n. 3-4, p. 689-702, jul. 1996.

VERSCHUEREN, J. Contrastive Pragmatics. In: ÖSTMAN, J.-O.; VERSCHUEREN, J. (Org.). Handbook of Pragmatics. Amsterdam: John Benjamins, 2016. Disponível online <https://benjamins.com/online/hop/articles/hop.20.con18>. Acesso em: 27 out. 2020 . 